\title{
FATORES DE RISCO E ASPECTOS FISIOPATOLÓGICOS ENVOLVIDOS NA NEFROPATIA DA HANSENÍASE: UMA REVISÃO INTEGRATIVA
}

\author{
RISK FACTORS AND PHYSIOPATHOLOGICAL ASPECTS INVOLVED \\ IN HANSENIASIS NEPHROPATHY: AN INTEGRATING REVIEW
}

\author{
Joice Holanda Dias ${ }^{1}$ \\ Kassandra Lins Braga ${ }^{2}$ \\ Renata Lívia Fonseca Moreira de Medeiros ${ }^{3}$
}

RESUMO: Objetivo: analisar na literatura científica as possíveis causas do acometimento renal na Hanseníase, partindo da seguinte questão norteadora: quais os principais fatores de risco e seus respectivos papéis na fisiopatologia da nefropatia da hanseníase? Método: trata-se de uma revisão integrativa da literatura realizada no ano de 2018. Para a sua produção, foram consultadas as bases de dados: Biblioteca Virtual em Saúde (BVS), Publicações Médicas (PubMed), Medline e biblioteca virtual Scientific Eletronic Library online (SCIELO), assim como foram empregados operadores booleanos e os Descritores em Ciência da Saúde (DECS): hanseníase, nefropatia, doença, rim e glomerulonefrite, em diferentes combinações. Resultados: a busca foi conduzida nas bases de dados selecionadas, com a pesquisa do descritor Hanseníase, isoladamente, e depois associado aos demais descritores. Em seguida, foram aplicados os filtros conforme os critérios de inclusão, sendo encontrados 42 artigos. Após exclusão das repetições e leitura dos resumos, foi possível identificar 5 estudos que convergiam para responder a questão norteadora levantada inicialmente. A análise dos artigos demonstra uma predominância de relatos de caso sobre pacientes com hanseníase que apresentaram rápido declínio da função renal. Tais casos são considerados raros na medicina, fato que pode ser explicado pela dificuldade em reconhecer os fatores de risco e obter o diagnóstico. Conclusões: Pacientes com hanseníase e fatores predisponentes para injúria renal, devem ter a função renal avaliada precocemente para diagnosticar lesões do parênquima. Como as manifestações clínicas costumam

\footnotetext{
1 Acadêmica do curso de Medicina pela Faculdade Santa Maria, Cajazeiras-PB. Email: joiceholandad@gmail.com.

Especialista em Preceptoria de Residência Médica pelo Hospital Sírio Libanês. Graduada em Medicina pela Faculdade de Medicina Estácio de Juazeiro do Norte. Docente da Faculdade Santa Maria. Coordenadora da Residência de Medicina de Família e Comunidade.

3 Doutora pela Faculdade de Ciências Médicas Santa Casa. Mestre em Enfermagem pela Universidade Federal da Paraíba (2013). Especialista em Saúde Pública pela Faculdade de Ciências Sociais e Aplicadas (2008). Graduada em Enfermagem pela Universidade Federal da Paraíba (2003). Docente da Faculdade Santa Maria.
} 
aparecer tardiamente, é preciso lançar mão de parâmetros laboratoriais sensíveis, e conforme mais estudos forem publicados a respeito da proteína quimiotática de monócitos urinários tipo 1 (MCP-1), esse biomarcador poderá contribuir para o diagnóstico e intervenção terapêutica no momento oportuno, evitando a perda da função renal do paciente.

Descritores: Doença; Glomerulonefrite; Hanseníase; Nefropatia; Rim.

ABSTRACT: Objective: to analyze in the scientific literature the possible causes of renal involvement in leprosy, based on the following guiding question: What are the main risk factors and their respective roles in the pathophysiology of leprosy nephropathy? Method: this is an integrative review of literature conducted in 2018. For its production, the following databases were consulted: Virtual Health Library (VHL), Medical Publications (PubMed), Medline and Scientific Eletronic Library (SCIELO), as well as Boolean operators and Health Science Descriptors (DECS): Leprosy, Nephropathy, Disease, Kidney and Glomerulonephritis in different combinations. Results: the search was conducted in the selected databases, with the leprosy descriptor search, alone, and later associated to the other descriptors. Afterwards, the filters were applied according to the inclusion criteria, being found 42 articles. After exclusion of the repetitions and reading of the abstracts, it was possible to identify 5 studies that converged to answer the guiding question. The analysis of the articles demonstrates a predominance of case reports on leprosy patients with a rapid decline in renal function. Such cases are considered rare in medicine, a fact that can be explained by the difficulty in recognizing the risk factors and obtaining the diagnosis. Conclusions: patients with leprosy and predisposing factors for renal injury should have early renal function evaluated to diagnose parenchymal lesions. As clinical manifestations tend to appear late, it is necessary to use sensitive laboratory parameters, and as more studies are published regarding urinary monocyte chemotactic protein type 1 (MCP-1), this biomarker can contribute to the diagnosis and therapeutic intervention in a timely manner, avoiding the loss of renal function of the patient.

Keywords: Disease; Glomerulonephritis; Kidney; Leprosy; Nephropathy. 


\section{INTRODUÇÃO}

A hanseníase é uma doença granulomatosa crônica causada pela Micobacterium leprae com prevalência mundial de 213.899 casos em 2014. A quantidade de casos novos no Brasil, segundo o Sistema de Informação de Agravos de Notificação (SINAN) da Secretaria de Vigilância em Saúde (SVS), em 2016, era de 25.218, 1696 (6,7\%) em menores de 15 anos e coeficiente geral de detecção de 12,2/100mil habitantes (DAHER et al, 2011) (OLIVEIRA et al, 2013) (OMS, 2016).

Embora haja uma tendência à estabilização dos coeficientes de detecção nacionais, a hanseníase ainda ocorre em índices elevados nas regiões norte, centrooeste e nordeste do Brasil, bem como, constitui um sério problema de saúde pública no país, sobretudo pela sua magnitude e alto potencial incapacitante (ROCHA et al, 2015).

Segundo dados do Ministério da Saúde (BRASIL, 2016), no Nordeste, especificamente no estado da Paraíba, o coeficiente de detecção, era de 9,63 casos/100 mil habitantes. Em 2010, nos municípios de Cajazeiras (sertão paraibano), Campina Grande (agreste paraibano) e na capital João Pessoa (litoral paraibano) foi apresentada a maior relevância epidemiológica do estado, com os coeficientes de detecção de 107,8 casos/100 mil habitantes, 20,8 casos/100 mil habitantes e 11,3 casos/100 mil habitantes, respectivamente (OLIVEIRA et al, 2013).

Alterações funcionais e morfológicas renais têm sido amplamente relatadas em pacientes hansenianos ao longo de décadas, tendo sido descritas pela primeira vez, ainda no início do século XX. Embora a exata patogênese que leva ao acometimento renal desses pacientes permaneça incerta, lesões do tipo: glomerulonefrite, distúrbios túbulo-intersticiais e nefroesclerose foram relatados por autópsias. Doença renal crônica também pode ocorrer e está associada principalmente à amiloidose (DAHER et al, 2011) (RIELLA, 2010).

Conforme estudo realizado por Rocha et al (2015), a partir do relacionamento probabilístico entre as bases do Sistema de Informações sobre Mortalidade (SIM) e 
SINAN, as doenças do aparelho geniturinário, principalmente a insuficiência renal crônica, aparecem em 2,6\% dos casos, dentre as causas de morte por hanseníase nas declarações de óbito (DO). Ficando atrás apenas das próprias sequelas da doença, que são responsáveis pelo óbito em 32,4\% dos casos; paradas cardiorrespiratórias, dentre outras causas mal definidas $(12,8 \%)$ e dos casos de septicemia $(9,2 \%)$.

De acordo com relatos descritos, o envolvimento renal pode estar presente em todas as formas da hanseníase, sendo mais frequente em pacientes multibacilares e nos que apresentam estados reacionais, principalmente eritema nodoso. Também, é perceptível maior prevalência em idosos. Dessa forma, é necessário investigar a presença de disfunção renal em pacientes com hanseníase, sobretudo naqueles que apresentam as condições acima mencionadas em associação (DAHER et al, 2011) (SILVA JUNIOR et al, 2015).

Considerando que a hanseníase, assim como suas manifestações espectrais, vêm sendo estudadas ao longo de séculos, o fato dessa doença ainda ocorrer de forma prevalente evidencia a necessidade de fortalecer as medidas de prevenção secundária e terciária vigentes. Nesse contexto, é imprescindível contribuir para a constante atualização da literatura médica sobre hanseníase, com fins de melhor compreensão da fisiopatologia de suas complicações, viabilizando abordagens terapêuticas mais eficazes.

Em face dos dados mencionados e das considerações feitas anteriormente, este estudo tem como objetivo analisar, nas produções científicas, as possíveis causas do acometimento renal na Hanseníase, partindo da questão norteadora: quais os principais fatores de risco e seus respectivos papéis na fisiopatologia da nefropatia da hanseníase?

\section{METODOLOGIA}

Trata-se de um estudo realizado por meio da revisão integrativa da literatura, uma vez que este método de pesquisa representa a mais ampla abordagem 
referente às revisões. Além disso, possibilita a síntese do estado do conhecimento sobre um determinado assunto, em diferentes lugares e momentos, mantendo-o atualizado e facilitando as mudanças na prática clínica como consequência da pesquisa (GERBER; ZAGONEL, 2013) (MENDES; SILVEIRA; GALVÃO, 2008) (SOUZA; SILVA; CARVALHO, 2010).

Conforme o percurso metodológico, seis etapas foram seguidas no desenvolvimento desta revisão. Primeira: definição da questão norteadora; segunda: delimitação dos critérios de inclusão e exclusão; terceira: escolha das bases de dados e busca das produções científicas; quarta: análise dos dados, quinta: discussão dos dados; sexta: síntese da revisão (MENDES, SILVEIRA; GALVÃO, 2008).

A primeira etapa foi definida a partir da formulação da questão norteadora: quais os principais fatores de risco e seus respectivos papéis na fisiopatologia da nefropatia da hanseníase?

Desse modo, foram elaborados os critérios de inclusão: estudos pertinentes à questão clínica da pesquisa, com texto completo disponível, publicados de 2013 a 2018 e escritos em português, espanhol ou inglês. Assim, excluíram-se os artigos publicados antes de 2013, artigos repetidos, pagos, e aqueles com variantes da temática que não atendessem à proposta desta revisão.

A busca foi conduzida nas bases de dados PubMed, Medline, biblioteca virtual Scientific Eletronic Library online (SCIELO) e Biblioteca Virtual em Saúde (BVS), com auxílio dos operadores booleanos e Descritores em Ciência da Saúde (DECS), em diferentes combinações: hanseníase, nefropatia, rim, doenças e glomerulonefrite. A coleta de dados ocorreu no ano de 2018.

\section{RESULTADOS}

A partir dos descritores e critérios escolhidos, a busca realizada nas bases de dados resultou em 42 artigos que abordavam o tema da pesquisa. Dentre estes, foram excluídos os que se repetiam e, após a leitura dos resumos, foi possível 
identificar 5 estudos que convergiam para responder à questão norteadora levantada inicialmente. A tabela 1 traz, de forma detalhada, a quantidade de artigos encontrada nas 4 bases de dados utilizadas.

Tabela 1 - Resultado da coleta de dados.

\begin{tabular}{|c|c|c|c|c|}
\hline DESCRITORES & BVS & SCIELO & Medline & PUBMED \\
\hline Hanseníase/ Leprosy & 8361 & $265^{\circ}$ & 639 & 861 \\
\hline AND Nefropatia/ Nephropathy & 3 & 1 & 1 & 7 \\
\hline $\begin{array}{ll}\text { AND } & \text { Glomerulonefritel } \\
\text { Glomerulonephritis }\end{array}$ & 7 & 2 & 3 & 3 \\
\hline $\begin{array}{l}\text { AND Rim AND Doença/ Kidney AND } \\
\text { Disease }\end{array}$ & 8 & 1 & 3 & 3 \\
\hline Total & 18 & 4 & 7 & 13 \\
\hline
\end{tabular}

A análise dos artigos demonstra uma predominância de relatos de caso sobre pacientes com hanseníase que apresentaram rápido declínio da função renal. Tais casos são considerados raros na medicina, fato que pode ser explicado pela dificuldade em reconhecer os fatores de risco e obter o diagnóstico.

De acordo com Meneses et al (2014) lesões renais podem estar presentes em mais de $72 \%$ das autópsias de pacientes com hanseníase, no entanto, apenas alguns pacientes apresentam proteinúria, edema e rápido declínio da função renal. Na maioria dos casos, não há manifestações clínicas.

As 5 publicações selecionadas trazem resultados que reforçam a gravidade da doença renal como potencial causa de morte em pacientes com hanseníase. $O$ artigo de Polito et al (2015) afirma que, nos casos em que o tratamento correto é empregado, as lesões renais causadas pela hanseníase são autolimitadas, predominantemente de origem glomerular e dificilmente evoluem com gravidade.

Dentre os 5 estudos, 3 foram realizados na Turquia, Espanha e Colômbia e 2 no Brasil. Apesar da diferença socioeconômica e cultural entre os países, os resultados e conclusões das publicações foram semelhantes, bem como as considerações a respeito da fisiopatologia e fatores de risco envolvidos. 
Nas tabelas 2 e 3 são ilustradas as principais características dos artigos analisados de acordo com periódico, ano, título, objetivo, principais resultados e conclusão.

Tabela 2 - Distribuição das publicações conforme ano e periódico científico.

PERIÓDICO

Renal Failure

Revista de la sociedad española de nefrología

Revista Infectio

Advances in Dermatology and Allergology

BMC Infectious Diseases

Fonte: dados da pesquisa, 2018.
ANO DE PUBLICAÇÃO

2015

2016

2015

2017

2014

Tabela 3 - Quadro-resumo dos estudos de acordo com título, objetivo, principais resultados e conclusão.

\section{TíTULO}

It is time to review concepts on renal involvement in leprosy: pre-and posttreatment evaluation of 189 patients.

\section{Glomerulone fritis por inmunocom plejos en un paciente com lepra.}

\section{OBJETIVO}

\section{PRINCIPAIS RESULTADOS E CONCLUSÃO}

Detectaram-se níveis elevados de creatinina em $45 \%$ dos pacientes no momento do manifestações clínicas e diagnóstico, $18 \%$ após 3 meses de tratamento laboratoriais de e 9\% após 8 meses. Os níveis foram altos doença renal em principalmente em homens, hipertensos, em pacientes com uso frequente de anti-inflamatórios não hanseníase antes esteroidais. Os resultados sugerem que as e após 0 tratamento com terapia multidrogas. lesões renais na hanseníase são atualmente leves, principalmente devido a aquisição da terapia multidrogas e ao tratamento eficaz dos episódios de reação.

Paciente de 25 anos deu entrada em serviço Relatar o caso de um paciente com hanseníase, de saúde com quadro de poliartrite simétrica aditiva inflamatória e eritema corporal. A partir da investigação clínica foi observado nível de glomerulonefrite mediada por creatinina elevado, associado a imunocomplexos e comprometimento poliarticular secundário ultrassonografia com sinais de nefropatia aguda. Após biópsia renal e das lesões de pele foi confirmado o diagnóstico de hanseníase multibacilar e eritema nodoso com comprometimento renal e poliarticular. É eritema nodoso. a importante fortalecer o ensino das doenças que dizem respeito à saúde pública para facilitar o diagnóstico e manejo das sequelas. 
Glomerulone Relatar o caso de fritis extracapilar y lepra: una asociación infrecuente. um paciente com hanseníase

associada glomerulonefrite extracapilar.

Paciente de 79 anos, diagnosticado com hanseníase aos 32, deu entrada em serviço de saúde com edema, hematúria e piora de função renal. Após biópsia, foi possível confirmar glomerulonefrite proliferativa a endocapilar e extracapilar. Esse quadro é raro e geralmente inicia associado à insuficiência renal aguda. $O$ tratamento de pacientes com hanseníase em hemodiálise é difícil, pois há pouca experiência e requer ajuste de doses.

Avaliar - Maior quantidade de cistos renais corticais, comprometimento renal em

Renal pacientes com involvement hanseníase,

in leprosy: Evaluation usando critérios of patients in Turkey

clínicos, bioquímicos ultrassonografi s.

\begin{tabular}{|c|c|}
\hline $\begin{array}{l}\text { Urinary } \\
\text { monocyte } \\
\text { chemotactic } \\
\text { protein-1 } \\
\text { (MCP-1) in } \\
\text { leprosy } \\
\text { patients: } \\
\text { increased for } \\
\text { risk kidney }\end{array}$ & $\begin{array}{l}\text { Avaliar o } \mathrm{MCP}-1 \\
\text { urinário e o o } \\
\text { estresse oxidativo } \\
\text { pela dosagem de } \\
\text { Malondialdeído e } \\
\text { correlacionar com } \\
\text { outros } \\
\text { marcadores de } \\
\text { doença renal. }\end{array}$ \\
\hline
\end{tabular}
hiperecogenicidade e níveis mais altos de creatinina, ureia e leucocitúria em comparação com os casos-controle. $\mathrm{O}$ acometimento renal na hanseníase, ao que tudo indica, está relacionado à quantidade de bacilos presentes no corpo. É importante que os pacientes sejam monitorados pela análise da urina, nível de creatinina e ultrassonografia para detectar o envolvimento renal precocemente.

Pacientes com hanseníase, sem tratamento prévio apresentaram maior excreção de albumina e maiores níveis de MCP-1 e Malondialdeído urinário em comparação com o grupo controle. Os níveis de MCP-1 foram particularmente elevados nos pacientes multibacilares, estando também associados a aumento do estresse oxidativo e excreção urinária de albumina. Dessa forma, esses pacientes têm grande risco de desenvolver manifestações clínicas de doença renal.

\section{DISCUSSÕES}

No que concerne aos fatores de risco envolvidos na nefropatia da hanseníase, os estudos citam: idade superior a 45 anos, classificação multibacilar e episódios de reação hansênica tipo II (eritema nodoso). De acordo com os resultados do artigo de Polito et al (2015), pacientes com hanseníase, em uso abusivo de Anti-inflamatórios não-esteroidais (AINES), e portadores de hipertensão 
apresentaram maiores níveis de creatinina em comparação com o grupo controle, sugerindo uma maior suscetibilidade dessa parte da amostra populacional.

De modo geral, não houve diferenças significativas no acometimento renal conforme o sexo. No entanto, segundo o artigo de Polito et al (2015), os homens da população estudada apresentaram maior nível de creatinina sérica, enquanto as mulheres manifestaram mais hematúria.

A bactéria Mycobacterium leprae não parece estar diretamente envolvida na formação das lesões renais, entretanto, sua presença tem sido detectada no parênquima renal de alguns pacientes. O estudo realizado por Ozturk et al (2017) sugere que a multibacilaridade é a causa de lesões renais mais graves e frequentes.

Assim como Ozturk et al (2017), Arriba et al (2016) destaca a forma lepromatosa (Classificação de Ridley-Jopling) ou multibacilar (classificação operacional) como fator de risco para amiloidose renal secundária, principalmente nos casos em que há episódios recorrentes de eritema nodoso ou úlceras cutâneas crônicas.

Embora os aspectos fisiopatológicos da nefropatia da hanseníase ainda não sejam inteiramente compreendidos, os autores concordam que as lesões glomerulares são causadas, provavelmente, por depósito de imunocomplexos. Além disso, o processo inflamatório crônico, em resposta à infecção, favorece o depósito de proteína amiloide, podendo levar a amiloidose secundária, defeitos na concentração e acidificação da urina, nefrite tubulointersticial, insuficiência renal aguda e glomerulonefrite (DÍAZ-RAMÍREZ, 2015).

O artigo de Meneses et al (2014) aborda o papel da proteína quimiotática de monócitos urinários tipo 1 (MCP-1) como biomarcador de lesão renal nos pacientes com hanseníase. Além disso, esse estudo traz duas hipóteses a respeito da fisiopatologia na formação das lesões renais: a primeira hipótese é que o depósito de imunocomplexos em pacientes multibacilares aumenta a carga de inflamação local, gerando injúria renal.

A segunda hipótese defende que a predominância de resposta imune tipo 2 é a responsável pela inflamação. Como sugerem os resultados desse estudo, o aumento de MCP-1 urinário está associado ao estresse oxidativo e maior chance de desenvolver doença renal; sabe-se, também, que essa proteína é fator crucial para o 
desenvolvimento de resposta imune tipo 2, dessa forma, a segunda hipótese é plausível.

Apesar de trazerem novas considerações a respeito dos fatores de risco e fisiopatologia da nefropatia da hanseníase, as publicações não são capazes de esclarecer completamente como se dá o acometimento renal dos pacientes. Isso reforça a necessidade de realizar mais estudos sobre essa temática, principalmente a respeito do MCP-1 urinário que parece ter um importante papel na origem da doença renal de pacientes com hanseníase, e que pode ser útil como biomarcador precoce para identificar pacientes de risco.

Os dois relatos de caso analisados descrevem situações nas quais o diagnóstico tardio e tratamento inicial inadequado contribuíram para a evolução grave dos pacientes. Segundo Díaz- Ramírez et al (2015), o fato da nefropatia da hanseníase ser uma hipótese diagnóstica pouco considerada, é um chamado ao fortalecimento do ensino das doenças de interesse à saúde coletiva nas escolas de medicina. Dessa maneira, seria possível estabelecer o diagnóstico e prevenir sequelas com maior eficácia.

\section{CONCLUSÕES}

Diante da morbidade que a doença renal pode acrescentar a hanseníase, a qual já é uma patologia incapacitante, é de grande importância compreender os fatores de risco e aspectos fisiopatológicos envolvidos nesse processo de adoecimento. Pacientes com hanseníase, especialmente da forma multibacilar, idosos, aqueles que já apresentaram episódios reacionais, hipertensos e os que, por algum motivo, necessitam do uso prolongado de AINES devem ter a função renal avaliada para diagnosticar lesões do parênquima precocemente.

Como as manifestações clínicas costumam ocorrer apenas nos casos mais graves, é preciso lançar mão de parâmetros laboratoriais sensíveis e, conforme mais estudos forem publicados a respeito do MCP-1 urinário, esse biomarcador poderá 
contribuir para o diagnóstico e intervenção terapêutica no momento oportuno, evitando a perda da função renal do paciente.

\section{REFERÊNCIAS BIBLIOGRÁFICAS}

ARRIBA, G. et al. Glomerulonefritis extracapilar y lepra: una asociación infrecuente. Nefrología (Madrid), v. 36, n. 3, p. 313-314, 2016.

BRASIL, Ministério da Saúde. PORTAL DA SAÚDE. Situação Epidemiológica da hanseníase no Brasil, 2016. [internet]. Disponível em:<http://portalsaude.saude.gov.br/index.php/oministerio/principal/leia-mais-o-ministerio/705-secretaria-svs/vigilancia-de-a-az/hanseniase/11298-situacao-epidemiologica-dados> Acesso em 14 de outubro de 2017.

DAHER, E. F. et al. Renal dysfunction in leprosy: a historical cohort of 923 patients in Brazil. Tropical doctor, v. 41, n. 3, p. 148-150, 2011.

DÍAZ-RAMÍREZ, Gabriel Sebastián; MORALES-PLAZA, Cristhian David; MATTA-CORTÉS, Lorena. Glomerulonefritis por inmunocomplejos en un paciente con lepra. Infectio, v. 21, n. 1, p. $51-55,2017$.

FARIA, L; SANTOS, L. A. C. Hanseniasis and its history in Brazil: the history of a "national scourge". História, Ciências, Saúde-Manguinhos, v. 22, n. 4, p. 1491-1495, 2015.

GERBER, V. K.Q; ZAGONEL, I. P. S. A ética no ensino superior na área da saúde: uma revisão integrativa. Revista Bioética, v. 21, n. 1, 2013.

MENDES, K. D. S; SILVEIRA, R. C. P; GALVÃO, C. M. de. Revisão integrativa: método de pesquisa para a incorporação de evidências na saúde e na enfermagem. Texto \& contexto enfermagem, v. 17, n. 4, 2008.

MENESES, G. C. et al. Urinary monocyte chemotactic protein-1 (MCP-1) in leprosy patients: increased risk for kidney damage. BMC infectious diseases, v. 14, n. 1, p. 451, 2014.

OLIVEIRA, V. M. et al. Levantamento epidemiológico da hanseníase no nordeste brasileiro durante o período de 2001 - 2010. Scire Salutis, Aquidabã, v.3, n.1, Out, Nov, Dez 2012, Jan, Fev, Mar 2013.

ORGANIZAÇÃO MUNDIAL DA SAÚDE. Estratégia global para Hanseníase 2016-2020: Aceleração rumo a um mundo sem hanseníase. [internet]. Disponível em: < http://apps.who.int/iris/bitstream/10665/208824/8/9789290225201-Portuguese.pdf> Acesso em 25 de outubro de 2017.

OZTURK, S.; OZTURK, T.; CAN, I. Renal involvement in leprosy: evaluation of patients in Turkey. Advances in Dermatology and Allergology/Postępy Dermatologii i Alergologii, v. 34, n. 3, p. 240, 2017.

POLITO, M.G. et al. It is time to review concepts on renal involvement in leprosy: pre-and posttreatment evaluation of 189 patients. Renal failure, v. 37, n. 7, p. 1171-1174, 2015.

RIELLA, M. C. Princípios de Nefrologia e Distúrbios Hidroeletrolíticos. Editora Guanabara, $5^{\text {a }}$ edição, 2010. 
ROCHA, M. C. N et al. Deaths with leprosy as the underlying cause recorded in Brazil: use of data base linkage to enhance information. Ciência \& saúde coletiva, v. 20, n. 4, p. 1017-1026, 2015.

SILVA JÚNIOR, G. B. et al. Amyloidosis and end-stage renal disease associated with leprosy. Revista da Sociedade Brasileira de Medicina Tropical, v. 43, n. 4, 2010.

SILVA JÚNIOR, G. B. et al. Leprosy nephropathy: a review of clinical and histopathological features. Revista do Instituto de Medicina Tropical de São Paulo, v. 57, n. 1, p. 15-20, 2015.

SILVA JÚNIOR, G. B. da; DAHER, E. F. Tropical diseases-associated kidney injury. Revista Brasileira de Clínica Médica, São Paulo, v. 11, n. 2, p. 155-64, 2013.

SOUZA, M.T. de; SILVA, M. D. da; CARVALHO, R. de. Revisão integrativa: o que é e como fazer. Einstein, v. 8, n. 1 Pt 1, p. 102-6, 2010. 